\title{
FROM OPEN GEOGRAPHICAL DATA TO TANGIBLE MAPS: IMPROVING THE ACCESSIBILITY OF MAPS FOR VISUALLY IMPAIRED PEOPLE
}

\author{
J. Ducasse ${ }^{a, b}$, M. Macé ${ }^{a, b}$, C. Jouffrais ${ }^{a, b}$ \\ ${ }^{a}$ University of Toulouse, IRIT, 118 Route de Narbonne, Toulouse, France - first.lastname@ irit.fr \\ ${ }^{\mathrm{b}}$ CNRS, IRIT, 118 route de Narbonne, Toulouse, France
}

Commission II, WG II/6

KEY WORDS: Tactile maps, tangible maps, visual impairment, open data, Human-Computer Interaction

\begin{abstract}
:
Visual maps must be transcribed into (interactive) raised-line maps to be accessible for visually impaired people. However, these tactile maps suffer from several shortcomings: they are long and expensive to produce, they cannot display a large amount of information, and they are not dynamically modifiable. A number of methods have been developed to automate the production of raised-line maps, but there is not yet any tactile map editor on the market. Tangible interactions proved to be an efficient way to help a visually impaired user manipulate spatial representations. Contrary to raised-line maps, tangible maps can be autonomously constructed and edited. In this paper, we present the scenarios and the main expected contributions of the AccessiMap project, which is based on the availability of many sources of open spatial data: 1/ facilitating the production of interactive tactile maps with the development of an open-source web-based editor; $2 /$ investigating the use of tangible interfaces for the autonomous construction and exploration of a map by a visually impaired user.
\end{abstract}

\section{1. "VIZUALISATION" OF SPATIAL INFORMATION BY VISUALLY IMPAIRED USERS}

Online maps have become widely used by sighted users, but they are not accessible to visually impaired users. The most accessible maps are tactile maps with outlines printed in relief. Those maps are called raised-line maps and provide visually impaired users with tactile cues that help to understand geographical data. In educational centers for blind and visually impaired people raised-line maps are commonly used during geography, mathematics, physics, etc., lessons, as well as during orientation \& mobility sessions. These maps are either embossed in a plastic sheet or printed on a special heat-sensitive paper that makes the ink swell when it is heated.

Designing a tactile map is a complex and time-consuming process that requires tactile graphics specialists called transcribers. According to the Braille Authority of North Canada $^{1}$, the transcriber must first select the information that will be presented on the tactile map, and possibly simplify or reorganize it so that the original map information may be spread over several tactile maps. Each element of the original map needs to be categorized into one of the four primary components (area, line, point or label) and will be transcribed with a specific outline or texture. The resulting graphic generally consists in one title, a north indicator, and the tactile map upon which Braille abbreviations are displayed. The Braille legend is usually presented on a separate sheet as illustrated in Figure 1.

Several studies have shown that visually impaired people could benefit from tactile maps. For example, Jacobson (1992) found that tactile maps provide visually impaired adults with a mental representation of the area that is represented. Espinosa et al. (1998) corroborated these results by demonstrating that visually

\footnotetext{
${ }^{1}$ http://www.brailleauthority.org/tg/web-manual/index.html
}

impaired participants who learned a route from a tactile map performed as well as visually impaired participants who learned the same route from direct experience, i.e. by walking it. However due to the lack of commercialized tactile maps it is difficult for the visually impaired to access spatial data (geography, geometry, technical drawings, etc.) and spatialized data (demographics, economics, etc.) but also to travel autonomously, which is likely to affect their personal and professional lives (Passini et al., 1988).

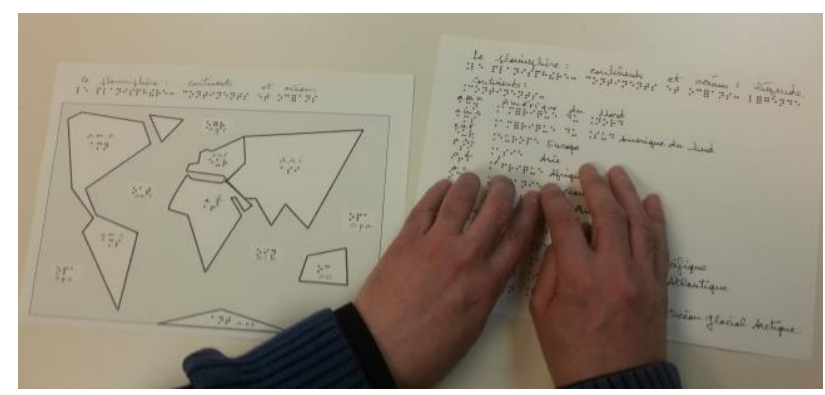

Figure 1. Example of a tactile map used by a visually impaired user during a geography lesson. The planisphere is simplified and presented on a sheet of paper. The legend is presented on a separate sheet.

Besides, tactile maps suffer from several restrictions. Firstly, Braille labels must be added to the map for describing its content. As Braille letters are large, abbreviations are placed on the map and explained on a separate legend, which forces the user to switch back and forth between the map and the legend, and disrupts the tactile reading process. Secondly, to avoid confusion and limit the cognitive load, it is not recommended to use more than five textures per map, which further limits the amount of information that can be rendered. Thirdly, a tactile map cannot be updated dynamically, and hence may present 
outdated information. Finally, current tactile maps are not interactive, which limits how students and teachers can use them.

To be more accessible for visually impaired people, in addition of being "tactile", it is essential that simple maps be autonomously and instantly accessible, i.e. without having to go through a manual transcription process that takes time. It should also provide fundamental functions such as zooming, panning, annotating, or choosing between different contents on the same image (e.g. on a single geographic map: demographic or weather data, historic facts).

\section{BETTER ACCESSIBILITY WITH INTERACTIVE AUDIO-TACTILE MAPS}

Parkes was the first to describe an augmented tactile map (Parkes, 1988). In this device, the tactile map was placed upon a tablet and its content rendered using audio output instead of Braille. Interactive audio-tactile maps allow avoiding the use of a Braille legend, but they also increase the amount of information that can be displayed. Indeed several pieces of information can be attached to a same point of interest, and retrieved using different gestures for example. Since the work of Parkes, other interactive maps have been developed that use different input and output modalities, but also different devices. Brock et al. (2013a) classified prototypes of interactive maps depending on the content, the sensory modalities and the technology that they rely on. For example, the Talking TMap (Miele et al., 2006), Touch the map! (A. M. Brock, 2013b) and the instant tactile-audio map (Wang et al., 2009a) are three prototypes of tactile audio-tactile interactive maps where tapping gestures provide the user with audio descriptions of tactile elements (see Figure 2 for example). The Talking TMap also investigated the use of tap gesture to access several layers of information. For example, tapping twice on a street "causes the system to speak the address ranges for the right and left sides of that particular block" and "the third tap announces the length of the block". In another study, Brock et al. (2014) designed gestural interaction techniques that enable a blind user to choose between different types of information, retrieving the names or detailed information about points of interest, or measuring the distance between them. In a follow-up study, Brock et al. (2015) demonstrated that, compared to classical raised-line maps, interactive tactile maps improve efficiency and user satisfaction in a map exploration task. One of the main limitations of interactive tactile maps is that they require the manual production of raised-line overlays, which is a timeconsuming process.

Other devices such as SpaceSence (Yatani et al., 2012) or TouchOverMap (Poppinga et al., 2011) rely on a touch sensitive surface only (without overlay). When the finger passes over an element, vibrational and vocal feedbacks are rendered. These devices allow visually impaired users to access geographical information or estimate a distance between different locations. As these prototypes do not require a tactile overlay, the users can also zoom and pan (Poppinga et al., 2011).

Similarly, haptic devices such as a joystick or a phantom device can be used by a visually impaired user to explore a virtual map

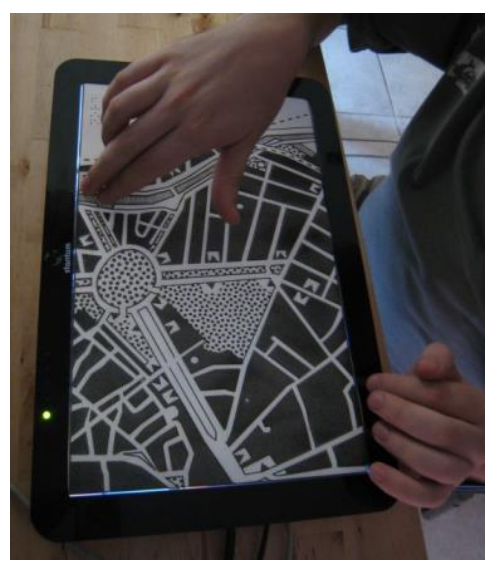

Figure 2. Touch the map! allows blind users to interact with a tactile map and retrieve names, distances and details using gestural interaction technique. Retrieved from (A. Brock et al., 2010).

(Kaklanis et al., 2013) (Simonnet and Ryall, 2013). However when haptic devices provide a single point of contact with the map, the exploration is highly sequential and rises demanding spatial and temporal mental processes to integrate the content of the map (Loomis et al., 1991). Rice et al. (2005) stated that "maintaining the relationship between the small areas being explored and the larger extent of mapped data is rather difficult without vision because of the significant memory loads".

Several technologies are promising to create a refreshable display suitable for interactive maps. One of the rare commercially available solution (HyperBraille ${ }^{2}$ ) consists in a matrix of $120 \times 60$ pins that can be moved up or down with piezoelectric crystals in order to display a tactile image. Zeng et al. (2014) used this device to enable blind users to explore, annotate and even zoom in or out. However such devices are relatively cumbersome but above all very expensive. A range of other technologies, including motors (Alexander et al., 2012), pneumatic (Yao et al., 2013) or electromagnetic (FriskenGibson et al., 1987) actuators (Nakatani et al., 2003), as well as hydraulic devices (Goulthorpe et al., 2001) are very promising to provide deformable screens. However, they are all in preliminary stages of development and are not available.

Currently, raised-line maps augmented with interactive auditory messages appear to be the more usable technology to provide visually impaired users with accessible maps. Indeed, complex spatial graphics can be explored with both hands and all the fingers. In addition, the device is cheap in comparison to refreshable displays. However, in order to enhance usability, it is essential to provide the users with tools that facilitate the production of raised-line maps with the corresponding interactive numerical content. We suggest that these tools should rely on the availability of numerous sources of spatial (including geographic) data that are free to use. OpenStreetMap ${ }^{3}$, for example, is a collaborative project that aims at creating a free and editable map of the world. These maps can be annotated with a variety of tags. Rice et al. (2013), for example, described several techniques to enhance map accessibility by adding crowdsourced information about temporary obstacles (Rice et al., 2013).

\footnotetext{
${ }^{2}$ Metec AG, Stuttgard, Germany

${ }^{3} \mathrm{http}$ ://www.openstreetmap.org
} 

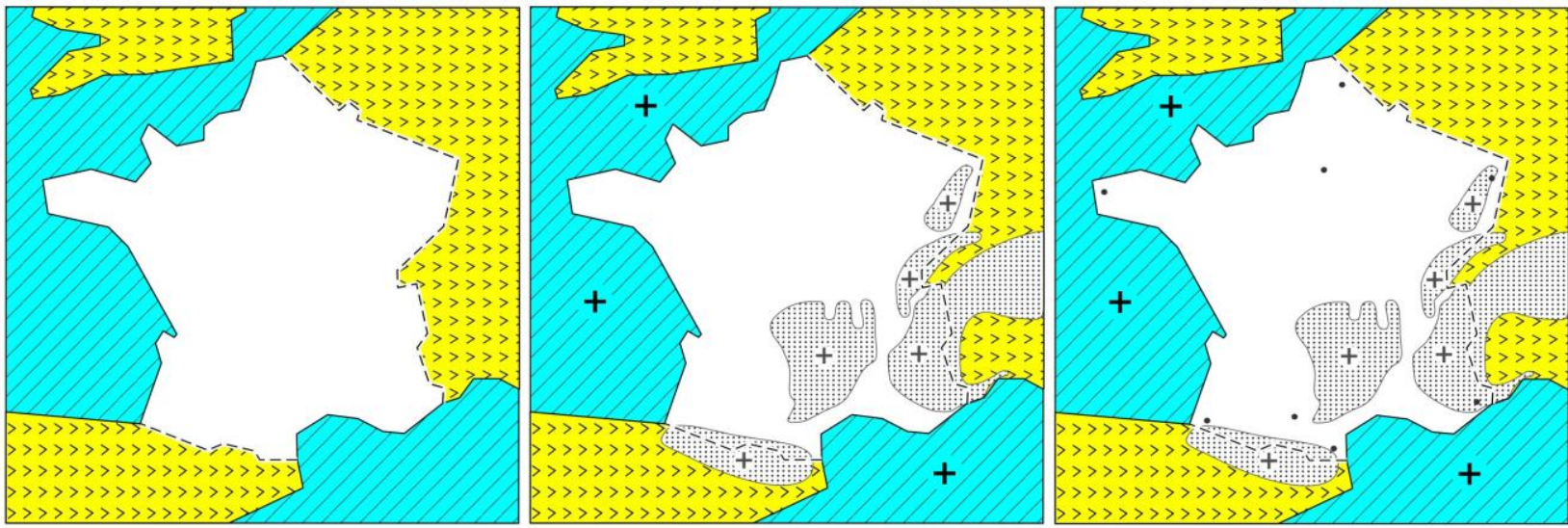

Figure 3. Example of three raised-line maps edited with the Open Street Map editor. The map on the left show the base map that has been used to create the two others maps by adding the ocean, sea and mountains (in the middle) and the main cities of France (on the right).

\section{COMPUTER-AIDED PRODUCTION OF INTERACTIVE TACTILE MAPS}

A few prototypes have been developed that enable visually impaired users to produce (interactive) audio-tactile maps on their own. Watanabe et al. (2014), for example, developed a web-based automated creation system that allows a visually impaired user to type in an address, and print the resulting tactile map. Although this project is really a progress in tactile map creation, it does not provide the user with the softcopy of the map needed for audio-tactile interaction. Wang et al. (2009) developed a system that automatically transcribes a map image into a tactile graphic, using text detection and graphic simplification algorithms. The resulting map is immediately printable with a tactile printer and may be placed upon a tablet that displays the softcopy of the map. By pressing the inserted symbols, users can get audio annotations. During the evaluation, all the visually impaired users managed to point out landmarks and trace routes, which prove that the automatic production of tactile map is a relevant method.

Other prototypes have been designed to help tactile graphics specialists quickly and easily produce tactile maps. This was in line with the work of Lobeen (2005) who interviewed seven orientation \& mobility instructors. All of them considered that it would be "beneficial ... if computer production of tactile maps was available in a simple, more accessible manner" and they all "consider using software designed specifically for the production of tactile maps". In (Takagi and Chen, 2014), a hand-drawn map was scanned by a sighted person, and automatically translated into a printable digital map using character and shape detection and classification. Götzelmann et al. (2014) designed a (semi) automatic generation of raised-line tactile maps than can be printed by $3 \mathrm{D}$ printers.

Unfortunately, to our knowledge, none of these prototypes is on the market or at least available for download. In addition, it is obvious that none of these methods allow dynamically editing the map, choosing the displayed information, defining the different feedbacks triggered by specific actions of the user, etc.

\section{TOWARDS TANGIBLE MAPS}

The main issue of interactive audio-tactile maps is that once the map is printed, its physical form cannot be edited. According to Ishii et al. (1997), tangible user interfaces "augment the real physical world by coupling digital information to everyday physical objects and environments". In our ongoing work, we suggest that a visually impaired user may add or (re)move tangible objects that represent areas, lines or points within the map. These objects, enhanced with non-visual interactions techniques, may improve accessibility to spatial digital data.

Using tangible objects may prove particularly relevant in supporting the (automatic) production of tangible maps. Tangible map prototypes were designed alongside the very first tangible user interfaces. GeoSpace is an interactive map of the MIT Campus designed for sighted users, where physical objects are used to pan or zoom by forcing the digital map to reposition itself (Ishii and Ullmer, 1997). Urp allows sighted urban planners to simulate wind flow and sunlight (Underkoffler and Ishii, 1999). It has been used to observe their consequences on models of building placed onto the tabletop. With the MouseHous Table, users can simulate several arrangements of urban elements such as streets and buildings (Huang et al., 2003). Paper rectangles were placed on the device and help to visualize the behaviour of pedestrians around the buildings. Similarly, ColorTable is a tool to help urban planners and stakeholders discussing urban changes (Maquil et al., 2008). In that project, a mixed-reality scene was created from the original map and the tangible objects that had been placed above it.

Tangible interfaces designed for visually impaired users are rare. To our knowledge there is no prototype that supports the production and edition of a geographic map. Schneider et al. (2000) developed a prototype in which vocal instructions guide a visually impaired user in placing rectangular magnets on a magnetic board until an entire route is built. This system is adapted to the creation of an itinerary but does not support the construction of complex maps. In addition, it has not been formally evaluated. McGookin et al. (2010) were the first to develop a prototype for the construction and exploration of tangible graphs by visually impaired users. In this prototype, a series of identical objects placed on a tabletop represented one series of data in a line plot. The evaluation showed that the prototype was efficient for the construction and comprehension of mathematical graphs by visually impaired users. 


\section{CURRENT AND FUTURE WORK}

AccessiMap ${ }^{4}$ is a research project that aims to improve map accessibility for the visually impaired. It brings together two research laboratories (IRIT, Toulouse, FR, and Telecom ParisTech, Paris, FR), a company that develops open software (Makina Corpus, Toulouse, FR), and an educational center for blind and visually impaired people (CESDV-IJA, Toulouse, FR).

\subsection{Scenarios and Expected Contributions}

Two major contributions of the AccessiMap project are expected: 1/ the development of a web-based authoring editor for the production of tactile graphics (overlays) and corresponding digital contents, which will be used on personal touch sensitive devices (tablets); 2 / the design and evaluation of a prototype of a tabletop tangible interface that enables the autonomous exploration of spatial data by visually-impaired users but also encourages the collaboration with sighted and other visually impaired users.

In collaboration with a number of specialized teachers and visually impaired persons we identified two scenarios that will serve as a guiding principle throughout the project. The first scenario concerns the production and the use of interactive tactile maps. Its implementation will lead to in-situ evaluations by teachers, tactile graphics specialists and visually impaired students. The second scenario explores the design space of nonvisual tangible maps: the prototypes will be evaluated in lab studies in order to design non-visual interactions and investigate the usability and the impact on spatial learning of visually impaired users.

Scenario 1: production and use of the audio-tactile map with raised-line overlays

Liz is an O\&M instructor. For her next lesson she has planned to make Paul discover a new itinerary, from Paul's house to Paul's new college. Using the web-based editor, Liz can quickly and easily produce a tactile map representing the itinerary. Before and during the lesson, Paul explores the tactile map laid upon a tablet and receives audio descriptions. He says that he better understands the whole spatial configuration of the neighborhood as well as the itinerary from home to college, but he misses fine details for many difficult intersections. Together they add vocal annotations to the map for helping Paul understanding these difficult points. Later on Liz edits the map in order to add the details that Paul needed. The overlay and the softcopy of the neighborhood are then usable by many other teachers and visually impaired students.

\section{Scenario 2: tangible interactions on and around a collaborative table \\ John is a blind student from the educational center. During the day he has walked a new route and recorded its trace using a dedicated application on his smartphone. Later in the day John launches the tabletop application, which guides him to reconstruct the route using tangible objects. Once the route is built, he can explore it with adapted non-visual interaction (including multitouch gestures, mid-air gestures, and objects manipulation). In addition, he can explore the whole neighborhood around the route. $\mathrm{He}$ can also listen to annotations recorded and shared by other users. The actuated (motorized) tangible objects provide him with multimodal}

\footnotetext{
${ }^{4}$ http://www.irit.fr/accessimap/
}

feedbacks (audio, vibration). The whole map is stored and available for other users. John and his friends can explore the area without depending upon a teacher or transcriber.

\subsection{Ongoing work: Development of an open-source web- based editor based on OpenStreetMap data}

In collaboration with Makina Corpus company, we are currently developing a web-based editor to facilitate the production of tactile maps by (semi)-automatically selecting and adapting OpenStreetMap data or an existing SVG file.

As we previously said, the content displayed on a tactile map differs from that of a visual map. Then the editor must allow the semi-automatic simplification of a visual map, but also the addition of mandatory elements, such as the presence of obstacles or the exact location of pedestrian crosswalks. OpenStreetMap data does not always meet these requirements but it can be adapted. Contents can be simplified with filters and simple geometrical transformations. Data can also be updated by adding new tags, which are adapted to the needs of visually impaired persons.

5.2.1 Participatory design to identify needs: During all the design steps, we are working closely with the professional transcribers and teachers of the educational center, especially to identify their needs and elaborate new functionalities.

For example, we are currently improving the interactive tactile map designed by Brock et al (2015) in order to better understand how it could be used during a geography lesson. This prototype includes a tactile overlay placed over a touch sensitive surface. Usages were observed during a geography lesson with a specialized teacher and her class of pupils aged between 10 and 12 years old. The aim of the lesson was to widen the students' knowledge of the geography of France and of the points of the compass. The students had to find pieces of information relative to a city: names of culinary specialities, accents, objects that they had handled during a previous lesson that represented a certain city, etc. To do so they first had to use a menu to select the corresponding type of data, and then had to locate the city. All of the participants showed a great interest in the device and almost immediately grasped how to use it.

After this lesson the first need that appeared was the creation of new overlays and corresponding digital contents in order to complete the activity with other notions (mountains, rivers, etc.). The observations allowed us to precisely specify the functions needed in the editor: for example the specialized teacher wants to create very specific maps that she will use conjointly with an O\&M instructor to work with the students on the means of transport at different geographic scales.

5.2.2 Functionalities: A number of functionalities are currently being investigated or developed:

1. Selecting the original file. To create a map, the user can either import a SVG file or select a geographic location. This allows the user to easily edit an existing map or start editing a new one without having to do it from scratch.

2. Selecting the degree of simplification of the map. This can be done by selecting filters (type and strength) and observing the resulting drawing in real time. The higher the strength is, the straighter the lines are and the less detailed the map is. 
3. Choosing the type of symbols to be used: Even though there are guidelines for the production of tactile graphics, the teachers and transcribers may choose specific patterns (such as plain or dotted lines, circles or triangles, thin or thick lines, etc.) to highlight particular features of the map (for example two different textures will be used to distinguish between old and recent mountains).

4. Adding new points, lines, circles or polygons. O\&M teachers often need to add particular point of interests, depending on the students' needs (traffic lines, pedestrian crossings, etc.). Geographic teachers also need to draw simplified areas to represent mountains or population densities for example (see Figure 3).

5. Editing or adding labels. It might be necessary to personalize the names of the map elements or to add new labels that are missing in the original map (the name of a building or a bus stop for example).

6. Selecting a layer of information. It is useful to be able to select a single layer of information in order to facilitate the edition of a category of elements (the streets or the bus stops for example). This can be done by selecting the layers that we want to display in a check-box menu (streets, bus stops, pedestrians crossings, points of interests, parks, etc.).

\section{Selecting or adding different pieces of information attached} to the same element.

8. Attaching an action listener to an element. The professionals must explicitly create interactive elements. The authoring tool allows attaching an action listener to each one on the map. For example, one will select a point of interest and declare that when a double-tap is performed on it, the associated audio file must be played. Elements are not interactive by default to minimize the number of unintentional selections when the user is exploring the map.

\subsection{Ongoing work: Development of a tabletop tangible interface for non-visual construction and exploration of a map}

5.3.1 Motivation: As we previously said, tabletop tangible interfaces have been developed for sighted users and they proved useful and efficient to dynamically manipulate spatial layouts. We will investigate how a non-visual tangible prototype could enable a visually impaired user to autonomously construct and explore a map, but also how it could foster a collaborative activity between a sighted teacher and visually impaired students or among a group of visually impaired students. This device could open new ways to interact with maps, but it will also serve as a support to address research issues concerning the usability of tangible interaction techniques and their impact on spatial learning of visually impaired users. We aim to fulfil the criteria that we previously listed:

- Interactivity: Tangible objects will be used to represent landmarks that are useful for understanding the spatial layout of the map (points of interests, buildings, streets, etc.). These objects can be used to initiate commands (e.g. adding a vocal annotation) but also to provide the user with audio feedbacks (e.g. speak the attached annotations). Other non-visual interactions based on gestures performed on and above the table are available. Similarly, they can provide the user with commands and feedbacks (for example, listening to digital annotations associated to the elements of the map). All these interaction techniques should enhance the number of functionalities (calculating a distance between two points, finding the shortest route, etc.), but also the users' satisfaction.

- Autonomous and instantaneous access: the different nonvisual interaction techniques that we just described should provide the user with the possibility to promptly build a simple map, without having to rely on a professional transcriber.

- Dynamic "visualization": One of the main issues of audiotactile maps is that it does not enable a blind user to zoom or pan. This limit can be overcome by constructing maps with tangible objects that the users can move, add or remove depending of the area that they want to explore. The tangible objects could reposition themselves to match the digital map that has been moved or zoomed by the user.

5.3.2 Design of tangible objects: Before developing the device, it is essential to study the design of the physical icons (phicons). McGooking et al. (2010) provide three guidelines relative to non-visual phicons: 1/ They should be physically stable to prevent them from being knocked over by the user; 2/ They should have irregular shape "so they can be placed on the table one way"; $3 /$ The system must provide awareness of phicon status to inform the user when a phicon is not detected.

We designed cylindrical objects with tactile cues for orientation. They are fillable with lead, which proved to be an efficient way to quickly modify their weight and make them stable. These objects are tagged and their position and current state (e.g. detected by the system) is continuously tracked by the system. In order to construct tangible maps, we also needed lines and areas. We added retractable reels on the objects. The end of the reel is magnetic and can easily be attached to another object. These connectable objects are used to "materialize" points, lines, and areas on the map (see Figure 4). We are currently evaluating the usability of these objects in our different scenarios.

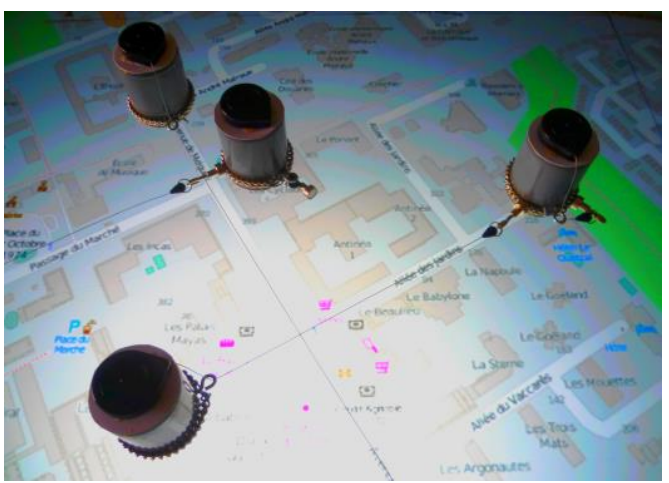

Figure 4. Tangible objects on the collaborative table. Here the objects represent either buildings or crossings. Their position and identity are monitored by the system. Streets are "materialized" using badge reels' strings. The objects are filled with lead to prevent the users from knocking them down when exploring the map. 
5.3.3 Towards actuated tangible objects: McGookin et al. (2010) suggest inserting "programmable microcontrollers into each phicon" so that they can communicate their own state. The tangible objects could also be actuated so that they could move autonomously until they reached a desired position, in order to construct a map, or to reposition itself after the user zoomed or panned.

We are currently prototyping similar objects with 3D printing techniques, which include an Arduino $\odot$ microcontroller, several sensors, and a motor. These objects communicate with the computer and can enhance non-visual interactions possibilities. They will provide the user with command selection as well as multimodal feedback as they can move, vibrate or play a sound.

\section{DISCUSSION}

In that position paper, we described how the accessibility of maps could be improved for visually impaired users. During brainstorming sessions and preliminary observations conducted with teachers and pupils of the educational center involved in the project, it appeared that both the authoring software and the collaborative table may modify the current practices and provide new tools for non-visual spatial learning. They also appeared to be more enjoyable than regular raised-line maps.

These two prototypes are still under development, but they will be evaluated soon in the lab, under controlled conditions. They will also be evaluated in-situ, within the educational center, by a geography teacher and an O\&M trainer. During these evaluations we will focus on the usability of the tools, but we will also investigate if and how they facilitate the learning of spatial data. In-situ evaluations will finally provide us with important feedback on usages.

Even though the AccessiMap project focuses on the accessibility of maps, we suggest that the results could broaden to the accessibility of tactile graphics in general. Indeed tactile graphics are widely used in educational centers (graphs, scientific schemas, geometry figures, etc.) and they present the same issues as those observed for interactive maps (lack of interactivity, difficulty to produce them, etc.).

\section{ACKNOWLEDGEMENTS}

We wish to thank all the participants of the AccessiMap project: our colleagues from the IRIT and Telecom ParisTech research laboratories, Makina Corpus, the LACII and the CESDV-IJA. We are particularly grateful to the teachers, transcribers and students for their time and suggestions.

\section{REFERENCES}

Alexander, J., Lucero, A., \& Subramanian, S. (2012). Tilt displays: designing display surfaces with multi-axis tilting and actuation. Proc. Mobile HCI, San Francisco, CA, USA, pp. 161-170.

Brock, A. M. (2013a). Interactive maps for visually impaired people : design, usability and spatial cognition.
Brock, A. M. (2013b). Touch the map!: designing interactive maps for visually impaired people. ACM SIGACCESS Accessibility and Computing, 105, pp. 9-14.

Brock, A. M., Truillet, P., Oriola, B., \& Jouffrais, C. (2014). Making gestural interaction accessible to visually impaired people. In Haptics: Neuroscience, Devices, Modeling, and Applications, pp. 41-48.

Brock, A. M., Truillet, P., Oriola, B., Picard, D., \& Jouffrais, C. (2015). Interactivity Improves Usability of Geographic Maps for Visually Impaired People. Human-Computer Interaction, 30(2), pp. 156-194.

Brock, A., Truillet, P., Oriola, B., \& Jouffrais, C. (2010). Usage of multimodal maps for blind people: why and how. In $A C M$ International Conference on Interactive Tabletops and Surfaces - ITS '10, Saarbruecken, Germany, pp. 247.

Espinosa, M. A., Ungar, S., Ochaita, E., Blades, M., \& Spencer, C. (1998). Comparing methods for introducing blind and visually impaired people to unfamiliar urban environments. Journal of Environmental Psychology, 18(3), pp. 277-287.

Frisken-Gibson, S. F., Bach-y-Rita, P., Tompkins, W. J., \& Webster, J. G. (1987). A 64-Solenoid, Four-Level Fingertip Search Display for the Blind. Proc. IEEE BME, 34(12), pp. 963-965.

Götzelmann, T., \& Pavkovic, A. (2014). Towards automatically generated tactile detail maps by 3D Printers for Blind Persons, in Computers Helping People with Special Needs, 8548, Paris, France, pp. 1-7.

Goulthorpe, M., Burry, M., \& Dunlop, G. (2001). Aegis hyposurface: The bordering of university and practice. Proc. of ACADIA, Buffalo, NY, pp. 334-349.

Huang, C., Do, E. Y., \& Gross, D. (2003). MouseHaus Table: a Physical Interface for Urban Design. 16th Annual ACM Symposium on User Interface Software and Technology, Vancouver, Canada, pp. 41-42.

Ishii, H., \& Ullmer, B. (1997). Tangible bits: towards seamless interfaces between people, bits and atoms. Proceedings of the ACM SIGCHI Conference on Human Factors in Computing Systems, Los Angeles, USA, pp. 234-241.

Jacobson, R. D. (1992). Spatial Cognition Through Tactile Mapping. Swansea Geographer, 29, 79-88.

Kaklanis, N., Votis, K., \& Tzovaras, D. (2013). Open Touch/Sound Maps: A system to convey street data through haptic and auditory feedback. Computers \& Geosciences, 57, pp. 59-67.

Lobben, A. (2005). Identifying the needs of tactile map makers. Proceedings of XXII International Cartographic Conference, Coruna, Spain.

Loomis, J. M., Klatzky, R. L., \& Lederman, S. J. (1991). Similarity of tactual and visual picture recognition with limited field of view. Perception, 20(2), pp. 167-177.

Maquil, V., Psik, T., \& Wagner, I. (2008). The ColorTable: a design story. Proceedings TEI'08, Bonn, Germany, pp. 97-104. 
McGookin, D., Robertson, E., \& Brewster, S. (2010). Clutching at straws. In Proceedings of the 28th international conference on Human factors in computing systems - CHI '10, Atlanta, Georgia, USA, pp. 1715 .

Miele, J. A., Landau, S., \& Gilden, D. (2006). Talking TMAP: Automated generation of audio-tactile maps using SmithKettlewell's TMAP software. British Journal of Visual Impairment, 24(2), pp. 93-100.

Nakatani, M., Kajimoto, H., \& Sekiguchi, D. (2003). Form Display with Shape Memory Alloy. Proc. ICAT, Tokyo, Japon, pp. 179-184.

Parkes, D. (1988). "NOMAD": An audio-tactile tool for the acquisition, use and management of spatially distributed information by partially sighted and blind persons. In A. Tatham \& A. Dodds (Eds.), Proceedings of Second International Conference on Maps and Graphics for Visually Disabled People, Nottingham, United Kingdom, pp. 24-29.

Passini, R., \& Proulx, G. (1988). Wayfinding without vision: An experiment with congenitally, totally blind people. Environment And Behavior, 20(2), pp. 227-252.

Poppinga, B., Magnusson, C., Pielot, M., \& Rassmus-Gröhn, K. (2011). TouchOver map: Audio-Tactile Exploration of Interactive Maps. In Proceedings of the 13th International Conference on Human Computer Interaction with Mobile Devices and Services - MobileHCI '11, Stockolm, Sweden, pp. 545-550.

Rice, M. T., Jacobson, R. D., Caldwell, D. R., McDermott, S. D., Paez, F. I., Aburizaiza, A. O., Qin, H. (2013). Crowdsourcing techniques for augmenting traditional accessibility maps with transitory obstacle information. Cartography and Geographic Information Science, 40(3), pp. $1-10$.

Rice, M. T., Jacobson, R. D., Golledge, R. G., \& Jones, D. (2005). Design considerations for haptic and auditory map interfaces. Cartography and Geographic Information Science, 32(4), pp. 381-391.

Schneider, J., \& Strothotte, T. (2000). Constructive exploration of Spatial Information by Blind Users. Proceedings of the fourth international ACM conference on Assistive technologies, Arlington, VA, USA, pp. 188-192.

Simonnet, M., \& Ryall, E. (2013). Blind Sailors' Spatial Representation Using an On-Board Force Feedback Arm: Two Case Studies. Advances in Human-Computer Interaction, 2013, pp. 1-6.

Takagi, N., \& Chen, J. (2014). Development of a computeraided system for automating production of tactile maps and its usability evaluation. In 2014 World Automation Congres, Kona, Hawaii, pp. 213-218.

Underkoffler, J., \& Ishii, H. (1999). Urp: a luminous-tangible workbench for urban planning and design. Proceedings of the SIGCHI Conference on Human Factors in Computing Systems, Pittsburgh, Pennsylvania, USA, pp. 386-383.

Wang, Z., Li, B., Hedgpeth, T., \& Haven, T. (2009a). Instant Tactile-Audio Map: Enabling Access to Digital Maps for
People with Visual Impairment. In Assets '09 Proceedings of the 11th international ACM SIGACCESS conference on Computers and accessibility, Pittsburgh, Pennsylvania, USA, pp. 43-50.

Wang, Z., Li, B., Hedgpeth, T., \& Haven, T. (2009b). Instant Tactile-Audio Map: Enabling Access to Digital Maps for People with Visual Impairment. In Assets '09 Proceedings of the 11th international ACM SIGACCESS conference on Computers and accessibility, Pittsburgh, Pennsylvania, USA, pp. 43-50.

Watanabe, T., Yamaguchi, T., Koda, S., \& Minatani, K. (2014). Tactile Map Automated Creation System Using OpenStreetMap. In K. Miesenberger, D. Fels, D. Archambault, P. Peňáz, \& W. Zagler (Eds.), Proceedings of ICCHP 2014, Paris, France, 8548, pp. 42-49.

Yao, L., Niiyama, R., \& Ou, J. (2013). Pneumatically actuated soft composite materials for shape changing interfaces. Proc. UIST, St Andrews, United Kingdom, pp. 13-22.

Yatani, K., Banovic, N., \& Truong, K. (2012). SpaceSense: representing geographical information to visually impaired people using spatial tactile feedback. In Proceedings of the 2012 ACM annual conference on Human Factors in Computing Systems - CHI '12, Austin, Texas, USA, pp. 415 - 424.

Zeng, L., Miao, M., \& Weber, G. (2014). Interactive Audiohaptic Map Explorer on a Tactile Display. Interacting with Computers, 27(4), pp.413-429. 